\title{
Dissociation of vertical and horizontal components of somesthetic orientation- localization during recovery from cortical damage: Implication regarding central associative functions
}

\author{
ROBERT B. GLASSMAN \\ Lake Forest College, Lake Forest, Illinois
}

\begin{abstract}
Blindfolded cats in which SII and adjacent areas had been ablated were tested for their ability to locate cutaneous stimuli as demonstrated by their bringing the mouth into contact with the stimulated point. During recovery, seven cats went through a phase in which they displayed dissociations of the vertical and horizontal components of the orientation-localization movement: either they moved the head downward before initiating any lateral movement or they turned towards the side of stimulation well before achieving accurate proximodistal localization. Hypotheses are offered about which aspects of anatomy and physiology are involved in proximodistal and lateral localization, and a simple mathematical model is given to suggest that one reason why such central associative functions in movement may have evolved is because they require fewer neurons than an alternative conceivable design.
\end{abstract}

Two reciprocally related behavioral phenomena unexpectedly were observed during an experiment designed to measure the effects of cortical ablations on speed and accuracy of the cutaneous orientationlocalization movement and on learned cutaneous behavior. These phenomena suggest that, in the organizational scheme for the normal orientation-localization response, there are distinguishable components, or functions.

Even some simpler reflexes require participation of higher levels of the nervous system. For example, in contact placing, a tactile stimulus to the surface of the cat's forelimb elicits a coordinated movement involving motion of the same limb in the direction of the stimulus. Physiological and behavioral studies suggest that this response depends on the overlapping SI and MI maps on the sigmoid gyrus (Amassian, Ross, Wertenbaker, \& Weiner, 1972; Glassman \& Glassman, 1977; Villablanca, Marcus, Olmstead, \& Avery, 1976; Welt, Aschoff, Kameda, \& Brooks, 1967).

Orientation towards stimulated points on the body surface also requires stereotyped stimulus-response relationships, but the neural wiring problem for orientation-localization is more complex than that for contact placing because the muscles needed to

\footnotetext{
This study was supported in part by a grant from the National Science Foundation. I thank Doris E. Cook and Jill R. Glassman for their excellent histological work. Correspondence should be addressed to: Robert B. Glassman, Department of Psychology, Lake Forest College, Lake Forest, Illinois 60045.
}

move the cat's face towards a stimulated point on the body are not, in general, near that point. Moreover, the relative contributions to the movement by members of the same set of muscles vary depending on which point is stimulated.

Behavioral and physiological data have indicated that the following brain areas are among those that participate in orientation-localization behaviors: tectum, reticular formation, substantia nigra, pons, lateral hypothalamus, ventroposterior thalamus, amygdala, basal ganglia, parietal cortex, frontal cortex (Fabre \& Buser, 1981; Feeney \& Wier, 1979; Glassman, Forgus, Goodman, \& Glassman, 1975; Greeley, Hagamen, Hagamen, \& Reeves, 1975; Heilman \& Watson, 1977; Marshall, 1978; Sprague, 1966; Stein, Magalhaes-Castro, \& Kruger, 1976; Trulson, 1976; Turner, 1973). However, the organization of the stimulus-response relationships and of the underlying neural functions is not fully understood.

Previous cortical ablation experiments have indicated that although the cat's postural reflexes and voluntary control of movement depend on the sigmoid gyrus (SI, MI), cutaneous orientationlocalization and also learned responsiveness to cutaneous stimuli show significant deficits only when anterior ectosylvian gyrus (SII) and subjacent orbitalanterior sylvian gyrus association cortex (insular cortex) are damaged (Glassman \& Glassman, 1977; also see Norrsell's, 1980, review). Because some questions lingered about possible contributions of other cortical areas to cutaneous sensation, another series of 
20 animals was studied, and a description of these follow-up results is in preparation. Seven of these animals, some of whose ablations were larger than intended, showed surprising dissociations of the vertical and horizontal components of the orientationlocalization movement. Because this interesting result is distinct from the rest, it is reported now.

\section{METHOD}

For the present purpose, it is sufficient to report the following methodological features. Three of the animals received unilateral cortical ablation in a single operation (Cats F7, F8, and F9); in three animals, unilateral ablations were enlarged in a sequence of operations (Cats F16, F19, and F24); and in one, bilateral damage was carried out in two stages (Cat F18). Using sodium pentobarbital general anesthesia, cortical ablations were usually accomplished by aspiration. However, in order to preserve a histological record of the earlier ablations, the ablations of proreus and orbital gyri were carried out in Cats F16 and F24, not by aspiration, but by sectioning these gyri off from the rest of the brain with a spatula while leaving the tissue in place and most of the pia intact.

Histological examination of celloidin-embedded, cresyl-violetstained sections, taken at $0.5-\mathrm{mm}$ intervals, has verified that in different cats there were varying locations and extents of damage to the parts of the sensorimotor cortex and to adjacent areas of the dorsal, lateral, and basal frontal cortex labeled in Figure 1. The drawings in Figure 2 are tracings of photographs of the gross specimens oriented to best show the damage. These drawings were refined on the basis of microscopic examination at intervals of $0.5 \mathrm{~mm}$ of cresyl-violet-stained sections and on the basis of the surgical notes. Because the landmarks in the brains of Cats F16 and F24 were distorted after the multiple ablations, to improve the readability of Figure 2 the original detailed drawings of damage for these two animals have been translated onto the corresponding landmarks of the respective mirror images of the other, undamaged hemisphere. The underlying claustrum, putamen, globus pallidus, and amygdala were also damaged in Cats F7, F8, F9, F16, and F24, as further described below.

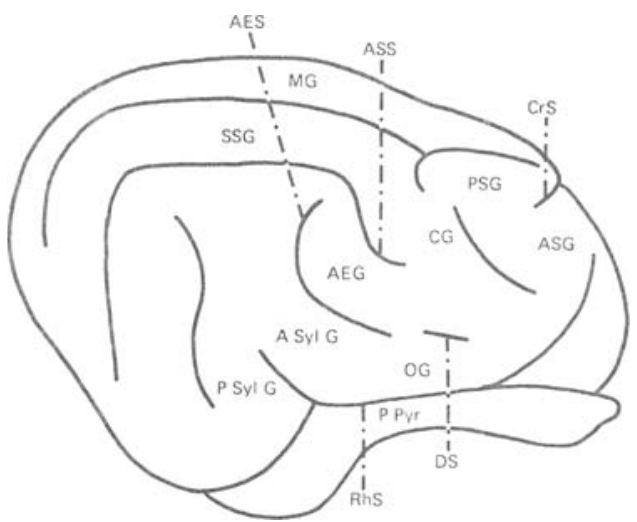

Figure 1. Basofrontolateral view of the right hemisphere of the cat's brain, reviewing the names of gyri and sulci involved in the cortical ablations. Abbreviations are as follows: ASG-anterior sigmoid gyrus; PSG-posterior sigmoid gyrus; SSG-suprasylvian gyrus; OG-orbital gyrus; AEG-anterior ectosylvian gyrus; A Syl G-anterior sylvian gyrus; CG-coronal gyrus; MG-marginal gyrus; P Pyr-prepyriform area; P Syl Gposterior sylvian gyrus; $\mathrm{CrS}$-cruciate sulcus; AES-anterior ectosylvian sulcus; DS-diagonal sulcus; ASS-anterior suprasylvian sulcus; RhS-rhinal sulcus.

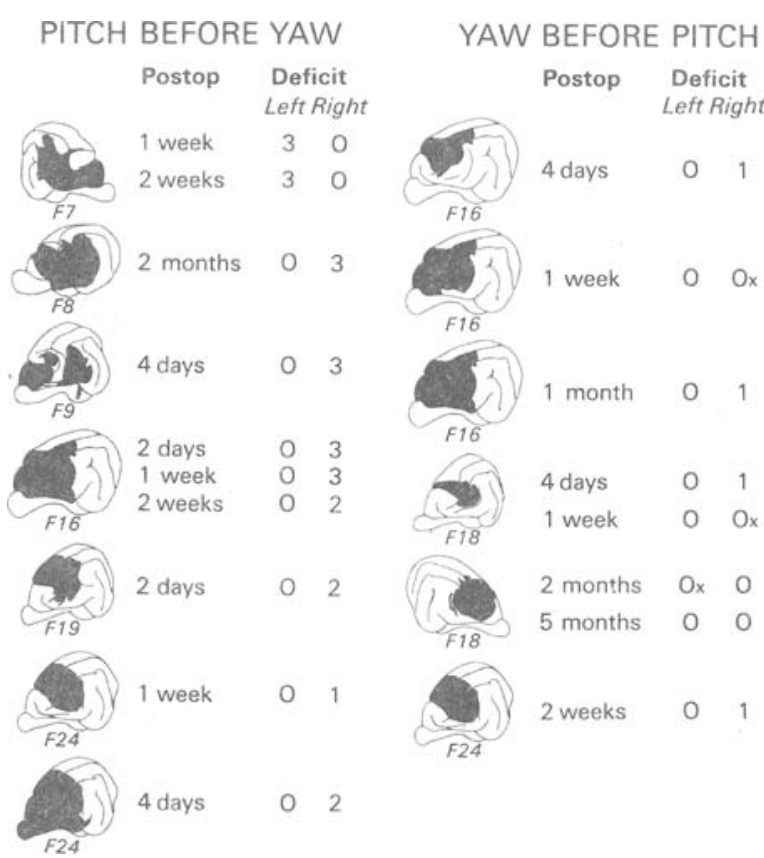

Figure 2. Reconstruction of cortical damage in each animal showing pitch before yaw or yaw before pitch, and indications of the deficit ratings and postoperative times at which these phenomena occurred. Animals receiving sequential ablations or showing both behavioral phenomena are illustrated more than once. A slight margin of white alongside a sulcus indicates that the damage did not extend all the way down to the bottom of the sulcus. Deficit ratings are on a 4-point scale in which 0 denotes no deficit on that side and 3 represents complete failure to turn towards the indicated side. The additional notation " $x$ " near some of the scores of 0 denotes performance recognized as slightly deficient only because of a small asymmetry. (All deficit scores were the same for proximal and distal stimulation except in four cases in which the indicated scores are proximal and the respective distal scores were as follows: Cat F18, left ablation, 1 week-left 0, right 1; Cat F18, right ablation, 2 months and 5 months-left 1, right 0; Cat F24, first ablation, 2 weeks-left 0 , right $0_{\mathbf{x}}$.)

Cats' behaviors were observed in a set of neurological tests of cutaneous, auditory, visual, postural, and motor abilities before surgery and on the following schedule postoperatively: 2 days, 4 days, 1 week, 2 weeks, 1 month, 2 months. All of these tests have been described in detail in earlier reports cited above. For the cutaneous orientation-localization test, the cats were blindfolded and then were repeatedly offered pieces of meat held in a long (30$\mathrm{cm}$ ) forceps that contacted various points on the body surface. Trials were usually initiated after the cat had assumed a ready posture of standing straight with muzzle oriented toward the experimenter. Control procedures ensured that the orientationlocalization response occurred to the cutaneous stimulus rather than to extraneous auditory, visual, olfactory, or temporal cues, and that the experimenter did not inadvertently initiate trials cued by variations in the cat's ready posture. The cat was tested for up to $15 \mathrm{~min}$ in this procedure, with emphasis on comparing left versus right and proximal versus distal responsiveness of the anterior half of the body. Such comparisons were sometimes facilitated by eliciting up to six responses to successive stimulations of different points at short intervals of 1 to $3 \mathrm{sec}$; during such procedures, the reward was withheld until the last response in the sequence. Qualitative notes were taken, and responsiveness was 
scored on a 4-point scale ranging from 0 (no deficit) to 3 (complete failure to turn even toward cutaneous pressure stimulation of the deficient side; additional details in the references already cited).

Normal cats respond in this test by reliably bringing the nose to within 1 or $2 \mathrm{~cm}$ of any stimulated point on the forelimb or ribs in a single, smooth, direct, brisk, and accurate motion. Responsiveness to the hindlimbs is more variable, but almost all normal cats clearly differentiate proximal and distal stimulation. In the normal cat, minor adjustments (by means of small head movements leading to vibrissal stimulation) of the initial localization response are more obvious with posterior than with anterior stimulation.

\section{RESULTS}

On a number of occasions of testing, six operated cats completed a downward movement immediately following stimulation of the forepaw contralateral to a cortical ablation before they initiated any lateral movement. In two of these six operated animals and in one other operated animal, the reciprocal phenomenon was also observed, at a later stage of recovery from the same ablation as well as following earlier ablations; these three cats responded briskly to stimulation by turning laterally well before achieving proximal-distal localization. Figure 2 illustrates the cortical damage in each of these animals and indicates the postoperative intervals at which each occurrence of movement dissociation was recorded. Also shown are the overall deficit scores at the time that these anomalies were recorded. It should be reemphasized that, although it is true that blind control procedures were not used, these phenomena were not sought and were not expected. The following analysis was not begun until long after all the data had been collected. For this analysis it is convenient to adapt the engineering terms "pitch," to describe the vertical component of movement, and "yaw," to describe the lateral rotation.

With the data arrayed as in Figure 2, it is evident that the pitch-before-yaw phenomenon is associated with a more severe cutaneous deficit than is the observation of yaw before pitch. Consistent with our earlier studies, the most severe and lasting deficits followed extensive damage to SII and insular cortex. In more detail, the pitch-before-yaw effect appeared as follows: stimulation of the deficient forepaw was followed immediately by a stimulus-bound lowering of the head to the table surface (slower than the response of a normal cat), and only then by small, diffuse searching movements left and right, which were random or which gradually drifted toward the deficient side. Control procedures in which the foreceps was not baited suggested that odor may have been a contributing cue in these very slow lateral searches.

Cats F16 and F24 are interesting in that each displayed pitch before yaw during the earlier postoperative period (up to 2 weeks and 1 week, respectively), but following additional recovery the opposite effect was seen during the next scheduled test. Thus, for example, by 2 weeks after the cortical ablation, most stimulations of the right forepaw of Cat F24 elicited an orientation-localization response that was aimed in the general direction of the right forepaw but that terminated as the cat bit several centimeters too high on the forceps that held the meat. On some occasions, this yaw-before-pitch effect was more pronounced; stimulation of the right forepaw elicited a simple lateral turning response, toward the proximal part of the limb, with no initial downward motion.

With both Cats F16 and F18, some instances of yaw-before-pitch mislocalizations were associated with a short-term learning effect. After several consecutive trials in which the paw was stimulated on the deficient right side, a subsequent stimulus to the proximal lateral surface of the right forelimb elicited an orientation towards the paw. Like Cat F24, Cat F16 was also sometimes observed to turn towards the proximal lateral right forelimb instead of towards the stimulated right forepaw; this occurred immediately after a sequence of trials in which accurate responses had been elicited to stimulation of the nondeficient proximal left forelimb. Consistent with these observations, this animal showed temporary improvement in responsiveness to the proximal or distal right forelimb after several trials in which there was stimulation of that same point. Thus, lacking adequate means for quickly determining proximal-distal location on the deficient side, the animal appeared to be "gambling" that successive stimuli would require the same degree of pitch. It is interesting that 1 week after the third operation (second picture in the right column of Figure 2) Cat F16 on two occasions responded to stimulation of the right forepaw first by turning left; then, checking this response, the cat turned to the right and, only after having done so, bent down to complete the localization.

The foregoing points about seven cats must be viewed in the context of the fact that there were 13 other cats (in this series of 20) in which dissociations were not observed. There were 40 operations on various subregions of frontal cortex in the 20 animals, and 29 of these 40 operations were followed by a cutaneous orientation-localization deficit. In these 29 cases, there were 11 cases of dissociation (including occurrences of both pitch before yaw and then yaw before pitch in 2 of these cases, for the total of 13 shown in Figure 2). The proportion of cases of dissociation was considerably higher than this for ablations following which there was an initial deficit rating of 2 or 3 and then recovery of at least one deficit rating point. Of 14 such cases, 8 displayed dissociations. Only 2 of the dissociation cases had an initial deficit rating of 1 (both of these were yaw before pitch), and the remaining case did not recover within 3 months from its initial deficit rating of 3 (pitch before yaw).

There is not a clear anatomical distinction between 
the dissociation cases and the "interesting" nondissociation cases just described. All 14 had varying damage, usually extensive, to coronal and anterior ectosylvian gyri. The posterior sigmoid and orbital gyri were also involved in cases of dissociation and cases of nondissociation. Varying degrees of accessory damage to the underlying basal ganglia also do not provide an anatomical basis for distinguishing clearly between dissociation and "interesting" nondissociation cases or between the two types of dissociation. Thus, while at least half of the claustrum and putamen-globus pallidus were ablated in dissociation Cats F7, F8, and F9-also in Cats F16 and F24, but probably only after the final, staged procedure in which the orbital gyrus was undercut with a spatula-the basal ganglia were intact in Cats F18 (both left and right) and F19 and the basal ganglia were very probably intact following the earlier (more dorsal) cortical ablations in Cats F16 and F24. Therefore, there was severe basal ganglia damage in five dissociation cases, four of which were pitch before yaw, and one of which showed both dissociations; there was little or no basal ganglia damage in six dissociation cases, of which one was pitch before yaw and one showed both dissociations. Three of the six "interesting" nondissociation cases were examined histologically; in two, the claustrum was $1 / 3-1 / 2$ ablated, with little or no damage to other basal ganglia, and in one, there was no basal ganglia damage. Of the remaining three "interesting" nondissociation cases, the surface pattern suggested severe damage to basal ganglia in one case, which included deep ablation of orbital gyrus, and none in the other two, in which the ablations were more dorsal. There was additional amygdala damage in four dissociation cases and probably in one nondissociation case.

The time needed to recover behaviorally by at least one deficit rating point was 1-2 months in almost all 14 cases of dissociation and "interesting" nondissociation having initial deficit ratings of 2 or 3.

\section{DISCUSSION}

Using a smooth, brisk, and accurate movement, an unoperated cat moves its face directly into proximity with a point stimulated on the body surface. The unexpected results described above, which were observed following some cases of damage to somatosensory cortex and adjacent regions, give a glimpse of what may be the underlying structure of this orientation-localization movement. The findings suggest that neural systems for pitch and yaw components of the movement are distinguishable. The fact that this distinction was revealed in some cats following cortical damage shows only that neural functioning at the cortical level has something to do with partially separable behavioral functions of pitch and yaw; the results do not show to what degree the pitch and yaw components may be organized in the cortex or to what degree the normal coordination between these components of movement is organized in the cortex. The possible contributing role of varying extents of accessory damage to underlying subcortical structures must also be acknowledged. The data do not tell us much in a direct way about possible anatomical localization of pitch and yaw. However, inferences about the neural underpinnings of behavior can often be made without precise anatomical definition of the lesions that yield a behavioral phenomenon (Meyer, 1958). The remainder of this discussion is such an analysis, which may help to guide further empirical attempts at localization or other understanding.

That there is not a global anatomical separation of the pitch and yaw functions is suggested by the findings that Cats F16 and F24 displayed pitch before yaw and then yaw before pitch at different stages of recovery from a given operation. Moreover, Cat F24 displayed pitch before yaw after each of two operations on the same hemisphere, while F16 showed yaw before pitch after each of three operations on a single hemisphere. These observations suggest that the dissociations are functions of damage to the same regions of cortex.

Because such varied ablations caused dissociations in some cats, it is puzzling that dissociations were not noticed in all the animals having comparable lesions. Perhaps this is because of small individual differences in the degree to which pitch-yaw coordination depends on the regions that were ablated, or because of this factor's interacting with the variations in the extent of ablations. It is also possible that a mitigating influence of carrying out some of the ablations in stages (Finger \& Stein, 1982) in some way made the occurrence of dissociation more or less likely in different cases. The present data do not enable choosing among these possibilities.

An additional important point in this regard is that observational methods can be reliable only so long as an observer who is not blind to the independent variable maintains a conservative bias. (Such conservatism is illustrated in one way by the use of a deficit rating scale that has only two levels intermediate between "no deficit" and "complete loss" of a directed lateral component of movement.) Thus, it is possible that some of the cats showed partial dissociations that were not noticed. All of the cases reported here were quite obvious to the unaided eye. It might be worthwhile to devise a method of replication in which frequent postoperative measurements would be taken using electronic movement sensors and supporting information-processing apparatus, to seek and quantify possible occurrences of partial dissociation.

In order to understand what the results may mean, 
it is helpful to work with two alternative, simple models of the possible structure of an orientationlocalization system.

(1) First, consider a conceptually simple but highly differentiated neural orientation-localization mechanism in which cutaneous inputs are mapped, perhaps monosynaptically, onto motor outputs in a one-to-one fashion. Such a mechanism would require a very large number of connections. In particular, a hypothetical organism in which each of $r$ muscle groups participated in moving the mouth to $s$ discriminable areas of the body surface would require a connection from each distinguishable area to each muscle group, that is, at least sr neural connections. With such a system, spatial summation of neural excitation would determine the direction of movement. It is conceivable that the ablated regions of cortex housed some portion of a special-purpose orientationlocalization system having properties like these and that loss of these cortical regions revealed a more primitive orientation mechanism. This hypothetical system might improve orientation-localization in a way analogous to that in which the sigmoid gyrus (SI and MI)-connected to the phylogenetically new, rapidly conducting lemniscal inputs and pyramidal outputs-participates in feedback adjustment of posture and voluntary limb movements. Logically, however, there appears to be somewhat less of a need for tight input-output coupling (Brooks, 1971) in orientation-localization, and empirically, orientationlocalization movements (e.g., bringing the mouth to a paw) take longer than do reflexive adjustments of a limb. Unless a strong adaptive advantage is conferred, it seems unlikely that natural selection would yield such an intricate special-purpose mechanism.

(2) The alternative model system is conceptually more complex in the sense that it requires an added hierarchical level. But this complication seems more than counterbalanced by an advantage of such a system. That is, many of the sr connections in the hypothetical mechanism described above could be eliminated in a nervous system that had a small number (a) of variably excitable central associative functions that determined spatial parameters of location and movement. In such a system, each distinguishable area and each muscle group would be connected to each associative functional subsystem; a unique output for each input could then be achieved with no more than as + ar spatially summating connections. Such a system might also be more readily shaped to greater accuracy or adapted to other purposes by natural selection or learning. (For more extensive discussions of the probable reasons for the evolution of hierarchical systems, their adaptive advantages, and symptoms of damage, see Jackson, 1884/1958, Pattee, 1973, Simon, 1969, Teitelbaum, 1982, Towe, 1971, and Glassman, 1978).
How many associative functions are necessary? The problem of reaching any point in a volume has been systematically studied by manufacturers of industrial "robot" arm devices. These devices must have any of a number of variations of a design in which one joint has two articulations and a second joint has a single articulation; each of the articulations is controlled by a subsystem of a computer (Engelberger, 1980). For one such example, picture an arm that is similar to that of primates, but which has a shoulder whose degrees of freedom are restricted to pitch and yaw and an elbow having only pitch. For a cat's mouth to be able to reach, not any point in a volume, but any point on its own body surface, it would be parsimonious for the muscles to be controlled by three central associative functions $(a=3)$ : one for pitch (proximal-distal), one for yaw (left-right), and one for controlling the central location of spinal bending to accomplish anteriorposterior localization.

While there do not seem to be separate anatomical regions for pitch and yaw, the data allow the conjecture that yaw is simply a function of the balance of inputs to the left and right hemispheres. Also consistent with this conjecture are the following two phenomena, observed in the present series of cats and also reported previously (Glassman \& Glassman, 1977).

During earlier postoperative tests, cats with the most severe unilateral deficits in orientation-localization (1) always overshot when turning towards a stimulated point on the nondeficient side and (2) sometimes turned the wrong way to stimulation of the deficient side. This latter symptom was usually seen when the hindlimb had been stimulated, but occasionally it occurred also to forelimb stimulation. Indeed, in one cat of the present series, this symptom was seen clearly even when the cutaneous stimulus was produced by an alligator clip placed on a digit pad of the left forepaw for several seconds; the cat hissed and turned in the wrong direction in an attempt to attack this noxious source. (I had previously checked this same moderately painful and nondamaging stimulus on my own finger.) Both of these observations suggest that a reciprocally inhibitory balance of contralateral and ipsilateral projections controls yaw, with the greater number of contralateral projections predominating in normal animals. Analogous inferences about reciprocal inhibition have been drawn from cases of lateralized neglect in humans (Kinsbourne, 1977).

This hypothesis also suggests a possible basis for recovery of yaw in a simple "rebiasing" of the system. Such rebiasing might involve a change as simple as increased concentration of a neurotransmitter, postsynaptic denervation hypersensitivity, or undifferentiated sprouting in denervated areas. Such physiological bases of rebiasing might be controlled 
by learning associated with exteroceptive inputs or by an internal monitoring of some sort.

Because no other aspect of topographic mapping is analogous to the brain's left-right differentiation into two hemispheres, the pitch function cannot have so simple a basis as yaw may have. It seems likely that pitch depends on some details of organization of the receptive fields of neurons that receive topographically ordered projections from the body surface. If yaw is sufficiently accounted for by a leftright hemispheric-balance function, then the orientationlocalization system would be adequately and parsimoniously constructed if the cutaneous receptive fields of its neurons were less reliable or were elongated horizontally but were more compressed and sharply differentiated vertically. Consistent with this hypothesis is the general behavioral observation that, although normal cats accurately localize points anteriorly, stimulation of the posterior half of the body elicits a more diffuse search. Thus, the third associative function (anterior-posterior localization of stimuli-response location of spinal bending) may be only a secondary property of the topographic maps.

Unfortunately for the present hypothesis, many reported somesthetic receptive fields, including those of cells in SII, are elongated vertically, rather than horizontally, along the limb (Andersson, 1962; Haight, 1972). However, localization may be a result of the organization of intersections and disjunctions of large receptive fields (Milner, 1970); therefore, population characteristics should be studied neurophysiologically with the present behavioral results in mind. In so doing, attention must be paid to all cells and not just those whose response characteristics are easiest to define or those whose receptive fields have the most reliable boundaries on all sides.

Since cats showing the pitch-before-yaw effect had extensive unilateral damage of SII and usually of insular cortex, it may be hypothesized that pitch is controlled in part (1) by remaining cortical maps on the damaged side, for example, SI (2) subcortically, and/or (3) by known ipsilateral projections to the remaining SII (Haight, 1972; Woolsey, 1958) and insular cortex (Albe-Fessard \& Besson, 1973). The occurrence of pitch before yaw in three cats in which the ablation included all or nearly all of SI and SIII (Cats F16, F19, F24 in Figure 2; see Dykes, 1978, for a further description of these maps) tends to rule out (1). There are a large number of possibilities for (2), suggested by the list, cited above, of regions that other researchers have found to be involved in orientation or lateralized neglect. To test for (3), it would be logical to add damage to the intact hemisphere in cats that were reliably displaying pitch before yaw. Two impediments to such a procedure are the fact that this phenomenon was a passing concomitant of recovery and the difficulty of maintaining and testing a preparation that might turn out to be bilaterally anesthetic. The observations reported here and in previous studies do not give an indication of what would happen in such a case. Unilateral cortical damage has led only to contralateral cutaneous orientation-localization deficits. Although some animals have shown ipsilateral decrements in learner cutaneous discrimination performance (smaller than the contralateral deficit), it is hard to say whether these bilateral deficits were due to a sensory loss or to a more general "confusion factor" of some sort (Glassman, Cook, \& Glassman, 1981; Glassman \& Glassman, 1977).

The foregoing considerations suggest a reason why the yaw-before-pitch deficit occurred following the pitch-before-yaw deficit in two cats. It must take less of the animal's remaining organizational resources to accomplish a relatively diffuse rebiasing of leftward versus rightward responsiveness to contralateral and ipsilateral left and right somatosensory inputs than to reorganize an appropriate pitch response using a possibly large number of remaining subcortical and/or cortical somatosensory receptive fields that are differentiated proximodistally. Thus, ipsilateral inputs may participate in mediating the early postoperative sluggish pitch response to stimulation of the deficient side. The comparative ease of rebiasing left versus right responsiveness then may lead to relatively rapid recovery of a yaw response that is brisker than the slow pitch response. Finally, depending on how many remnants remain of brain regions whose cells both are available for food-motivated orientation-localization and have receptive fields that are differentiated proximodistally, brisk, accurate, and reliable pitch recovers to a greater or lesser degree.

\section{REFERENCES}

Albe-Fessard, D., \& Besson, J. M. Convergent thalamic and cortical projections-The nonspecific system. In A. Iggo (Ed.), Handbook of sensory physiology (Vol. 2) Somatosensory system. New York: Springer-Verlag, 1973.

Amassian, V. E., Ross, R., Wertenbaker, C., \& Weiner, H. Cerebellothalamocortical interrrelations in contact placing and other movements in cats. In T. L. Frigyesi, E. Rinvik, \& M. D. Yahr (Eds.), Corticothalamic projections and sensorimotor activities. New York: Raven Press, 1972.

Andersson, S. A. Projection of different spinal pathways to the second somatic sensory area in cat. Acta Physiologica Scandinavica, 1962, 56, Suppl. 194, 1-74.

Brooks, V. B. Tight input-output coupling. Neurosciences $R e-$ search Program Bulletin, 1971, 9, 51-56.

DYKes, R. W. The anatomy and physiology of the somatic sensory cortical regions. Progress in Neurobiology, 1978, 10, 33-88.

ENGELBERGer, J. F. Robotics in practice: Management and applications of industrial robots. London: Kogan Page, 1980.

FAbre, M., \& Buser, P. Effects of lesioning the anterior suprasylvian cortex on visuo-motor guidance performance in the cat. Experimental Brain Research, 1981, 41, 81-88.

Feeney, D. M., \& Wier, C. S. Sensory neglect after lesions of substantia nigra or lateral hypothalamus: Differential severity 
and recovery of function. Brain Research, 1979, 178, 329-346.

Finger, S., \& Stein, D. G. Brain damage and recovery: $R e$ search and clinical perspectives. New York: Academic Press, 1982.

Glassman, R. B. The logic of the lesion experiment and its role in the neural sciences. In S. Finger (Ed.), Recovery from brain damage: Research and theory. New York: Plenum Press, 1978.

Glassman, R. B., Cook, D. E., \& Glassman, H. N. Prefrontal lesions and cutaneous responsiveness of cats: Learned discrimination, delayed response, orientation-localization, and transfer. Physiology \& Behavior, 1981, 26, 107-116.

Glassman, R. B., Forgus, M. W., Goodman, J. E., \& Glassman, H. N. Somesthetic effects of damage to cats' ventrobasal complex, medial lemniscus, or posterior group. Experimental Neurology, 1975, 48, 460-492.

Glassman, R. B., \& Glassman, H. N. Distribution of somatosensory and motor behavioral function in cat's frontal cortex. Physiology \& Behavior, 1977, 18, 1127-1152.

Greeley, H. P., Hagamen, S. J., Hagamen, W. D., \& ReEves, A. G. Bilateral sensory neglect following midsaggital reticular formation lesions in cats. Brain Behavior and Evolution, 1975, 12, 57-74.

Grobstein, P., Comer, C., \& Kostyk, S. K. Frog prey capture behavior: Between sensory maps and directed motor output. In J.-P. Ewert, R. R. Capranica, \& D. J. Ingle (Eds.), Advances in vertebrate neuroethology. New York: Plenum, 1983, in press.

HAIGHT, J. R. The general organization of somatotopic projections to SII cerebral neocortex in the cat. Brain Research, 1972, 44, 483-502.

Heilman, K. M., \& Watson, R. T. Mechanisms underlying the unilateral neglect syndrome. In E. A. Weinstein \& R. P. Friedland (Eds.), Advances in neurology (Vol. 18) Hemiinattention and hemisphere specialization. New York: Raven Press, 1977.

Jackson, J. H. Evolution and dissolution of the nervous system (1884). Reprinted in J. Taylor (Ed.), Selected writings of John Hughlings Jackson (Vol. 2). London: Staples Press, 1958.

Kinsbourne, M. Hemi-neglect and hemisphere rivalry. In E. A. Weinstein \& R. P. Friedland (Eds.), Advances in neurology (Vol. 18) Hemi-inattention and hemisphere specialization. New York: Raven Press, 1977.

Marshall, J. F. Comparison of the sensorimotor dysfunctions produced by damage to lateral hypothalamus or superior colliculus in the rat. Experimental Neurology, 1978, 58, 203-217.

MEYER, D. R. Some psychological determinants of sparing and loss following damage to the brain. In H. F. Harlow \& C. N. Woolsey (Eds.), Biological and biochemical bases of behavior. Madison: University of Wisconsin Press, 1958.

Milner, P. M. Physiological psychology. New York: Holt, Rinehart \& Winston, 1970.
Norrsell, U. Behavioral studies of the somatosensory system. Physiological Reviews, 1980, 60, 327-354.

PatTeE, H. H. (Ed.). Hierarchy theory. New York: Braziller, 1973.

Simon, H. A. The architecture of complexity. In H. A. Simon, The sciences of the artificial. Cambridge, Mass: M.I.T. Press, 1969.

Sprague, J. M. Interaction of cortex and superior colliculus in mediation of visually guided behavior in the cat. Science, 1966, 153, 1544-1547.

Stein, B. E., Magalhaes-Castro, B., \& Kruger, L. Relationship between visual and tactile representations in cat superior colliculus. Journal of Neurophysiology, 1976, 39, 401-419.

Teitelbaum, P. Disconnection and antagonistic interaction of movement subsystems in motivated behavior. In A. R. Morrison \& P. L. Strick (Eds.), Changing concepts of the nervous system. New York: Academic Press, 1982.

Towe, A. L. Sensory-motor organization and movement. Neurosciences Research Program Bulletin, 1971, 9, 40-51.

Trulson, M. E. Biological bases for the integration of appetitive and consummatory grooming behaviors in the cat: A review. Pharmacology, Biochemistry, and Behavior, 1976, 4, 329-334.

TURNER, B. H. Sensorimotor syndrome produced by lesions of the amygdala and lateral hypothalamus. Journal of Comparative and Physiological Psychology, 1973, 82, 37-47.

Villablanca, J. R., Marcus, R. J., Olmstead, C. E., \& Avery, D. L. Effects of caudate nuclei or frontal cortex ablations in cats: III. Recovery of limb placing reactions, including observations in hemispherectomized animals. Experimental Neurology, 1976, 53, 289-303.

Welt, C., Aschoff, J. C., Kameda, K., \& Brooks, V. B. Intracortical organization of cat's motorsensory neurons. In M. D. Yahr \& D. P. Purpura (Eds.), Neurophysiological basis of normal and abnormal motor activities. New York: Raven Press, 1967.

Woolsey, C. N. Organization of somatic sensory and motor areas of the cerebral cortex. In H. F. Harlow \& C. N. Woolsey (Eds.), Biological and biochemical bases of behavior. Madison: University of Wisconsin Press, 1958.

\section{NOTE}

1. Grobstein, Comer, and Kostyk (1983) have devised a model similar in some ways to that described here, on the basis of data regarding frogs' orienting behaviors after midbrain lesions. (Note added in proof.)

(Manuscript received July 23, 1982;

revision accepted for publication January $14,1983$. ) 